\title{
Evaluating the stability of atmospheric lines with HARPS ${ }^{\star}$
}

\author{
P. Figueira, F. Pepe, C. Lovis, and M. Mayor
}

\author{
Geneva Observatory, University of Geneva, 51 Ch. des Maillettes, 1290-Sauverny, Switzerland \\ e-mail: pedro.figueira@unige.ch
}

Received 7 January 2010 / Accepted 17 February 2010

\begin{abstract}
Context. When searching for extrasolar systems using the radial velocity technique, the need for high-precision measurements implies that a precise wavelength calibration is required. The choice of the calibrator is a particularly important open question in the infra-red domain, where precision and achievements remain inferior to those in the optical.

Aims. We investigate the long-term stability of atmospheric lines as a precise wavelength reference and analyze their sensitivity to different atmospheric and observing conditions.

Methods. We use HARPS archival data for three bright stars, Tau Ceti, $\mu$ Arae, and $e$ Eri, which span 6 years and include highcadence measurements performed over several nights. We cross-correlate this data with an $\mathrm{O}_{2}$ mask and evaluate both radial velocity and bisector variations to a photon noise level of $1 \mathrm{~m} / \mathrm{s}$.

Results. We find that the telluric lines in the three data-sets are stable down to $10 \mathrm{~m} / \mathrm{s}(\mathrm{rms})$ over the 6 years. We also show that the radial velocity variations can be modeled by simple atmospheric models, yielding a final precision of $1-2 \mathrm{~m} / \mathrm{s}$.

Conclusions. The long-term stability of atmospheric lines was $10 \mathrm{~m} / \mathrm{s}$ over six years, in spite of atmospheric phenomena. Atmospheric lines can be used as a wavelength reference for short timescale programs, yielding a precision of $5 \mathrm{~m} / \mathrm{s}$ without any correction. A higher precision, of $2 \mathrm{~m} / \mathrm{s}$, can be reached if the atmospheric phenomena are corrected for using the simple atmospheric model described, making it a very competitive method even on long timescales.
\end{abstract}

Key words. atmospheric effects - instrumentation: spectrographs - methods: observational - techniques: radial velocities planetary systems

\section{Introduction}

The discovery by Mayor \& Queloz (1995) of a hot Jupiter orbiting 51 Peg by means of radial velocity (RV) measurements inspired the ongoing search for extrasolar planets. This breakthrough was achievable only by using very precise wavelength calibration systems. Both the Th-Ar emission lamp with the cross-correlation function (CCF) method (Baranne et al. 1996), and the $\mathrm{I}_{2}$ cell with the deconvolution procedure (Butler et al. 1996), were extensively used to detect planets by RV measurements. Technological developments have enabled more advanced spectrographs to be built, such as HARPS (Mayor et al. 2003), and reduction and analysis methods have been perfected through the years (Lovis \& Pepe 2007). To this day, HARPS delivers the most precise RV measurements, with sub-m/s precision, which has allowed a succession of ground-breaking detections of the lightest planets known (Lovis et al. 2006; Mayor et al. 2009).

Given the proven stability of these two well-established wavelength references, little investigation was performed of viable alternatives. With time, RV measurements have become possible in the infra-red (IR) domain, where wavelength calibration remains in its infancy and no method has established itself as the paradigm.

In our attempt to measure RV with CRIRES at a very early stage of the instrument's life, we used atmospheric lines as wavelength reference (Huélamo et al. 2008). Figueira et al. (2010)

* Based on observations taken at the $3.6 \mathrm{~m}$ telescope at La Silla. describe an improved data reduction in which a precision of $5 \mathrm{~m} / \mathrm{s}$ on a timescale of one week is reached. This result was obtained in a RV standard star using $\mathrm{CO}_{2}$ lines as wavelength reference.

The usage of telluric lines as a precise wavelength reference dates back to the first attempts to achieve precise RV measurements, by Griffin \& Griffin (1973), for Arcturus and Procyon. Balthasar et al. (1982), Smith (1982), and Caccin et al. (1985) also explored adopting atmospheric lines as a viable alternative to wavelength calibration. Using $\mathrm{O}_{2}$ lines, these authors demonstrated that a precision of $5 \mathrm{~m} / \mathrm{s}$ was possible. This result is particularly significant because they used different RV determination methods (different instrumentation, different line fitting approaches, etc.). Snellen (2004) used the same principle on $\mathrm{H}_{2} \mathrm{O}$ lines and reached a precision of 5-10 m/s in UVES data. In light of these results, we ask the two following questions:

- What is the stability of atmospheric lines over long timescales?

- What is the sensitivity of atmospheric lines to different observing conditions?

To answer these two questions, we analyzed HARPS archival data, which spans 6 years. The high internal stability of HARPS leads to an unequalled precision in RV measurements. The $\mathrm{RV}$ variations of atmospheric lines can then be compared with the very precise wavelength calibration provided by Th-Ar. In this paper, we address both of the questions given above and assess the suitability of atmospheric lines for wavelength calibration. 
Table 1. The summary of the data set properties for the stars used in this paper. Note that the $\mathrm{S} / \mathrm{N}$ is calculated at the center of order 60 .

\begin{tabular}{lccccc}
\hline \hline Target & \# of observations & \# of days with observations & $\overline{\text { \#observations/day }}$ & Time span [d] & $\overline{\mathrm{S} / \mathrm{N}}$ \\
\hline Tau Ceti & 5270 & 110 & 47.9 & 2308 & 260 \\
$\mu$ Ara & 2868 & 117 & 24.5 & 2303 & 176 \\
$e$ Eri & 1527 & 104 & 14.7 & 2217 & 316 \\
\hline
\end{tabular}

The paper is structured as follows. In Sect. 2, we describe HARPS and the datasets used in our investigation. Section 3 describes the principles of our method and the data reduction. The results are presented in Sect. 4 and discussed in Sect. 5. We conclude in Sect. 6 with the lessons to learn from this campaign.

\section{Observations}

\subsection{Using HARPS as an "absolute reference"}

HARPS (Mayor et al. 2003) is a fiber-fed cross-dispersed echelle spectrograph installed at the $3.6 \mathrm{~m}$ telescope in La Silla. The main dispersion is provided by an R4 echelle grating in a Littrow configuration. The orders are then dispersed in a direction perpendicular to the dispersion direction by a grism and imaged by a $2 \times 2 \mathrm{k} 4 \mathrm{k}$ CCD mosaic. This optical design creates 72 orders that span the whole optical range from 3785 to $6915 \AA$. The spectral resolution was measured to be $R=110000$ and the mean dispersion $0.015 \AA / p x l(820 \mathrm{~m} / \mathrm{s})$. The sampling is 3.3 pixel per resolution element. The instrument is located in a vacuum vessel to avoid spectral drift caused by temperature and air pressure effects, which are kept below $0.01 \mathrm{~K}$ and $0.01 \mathrm{mbar}$, respectively. A Th-Ar emission lamp is used for wavelength calibration.

The very high stability of HARPS allows a precision of $1 \mathrm{~m} / \mathrm{s}$ to be reached routinely. When a precision better than $1 \mathrm{~m} / \mathrm{s}$ is required, a second channel can be used to image the Th-Ar lamp simultaneously with the science target. The proven intrinsic IP stability of HARPS permits us to study spectral line profile variations as well, which can be done using the well-know bisector technique (BIS) on the cross-correlation function (CCF). We note that long-term precision of HARPS was found to be better than $1 \mathrm{~m} / \mathrm{s}$ (see previous references), making this study possible for the first time to this level of precision.

\subsection{The selection of targets}

To perform our study, we selected targets bright enough to allow us to reach a photon noise of $\sim 1 \mathrm{~m} / \mathrm{s}$ on the atmospheric lines position error in individual exposures. Out of these we selected stars which had a high number of points $(>1000)$, covered a long time span and had, preferably, a very high cadence of measurements. We payed particular attention to stars followed in asterosismology campaigns. These campaigns, aiming at studying the stellar RV variations on a timescale of hours to days, followed the star across the sky throughout several consecutive nights.

By applying the mentioned criteria we pinpointed Tau Ceti (HD 10700), $\mu$ Ara (HD 160691) and $e$ Eri (HD 20794) as the most suitable targets for our tests. The details on the relevant properties of the data sets are provided in Table 1. Tau Ceti and $\mu$ Ara were followed in asterosismology campaigns, of 3 and 8 days, respectively. It is important to note that the beginning of data acquisition on these targets goes back to 2003, ensuring a long time span.

Our objective was simply to provide for a strong background against which the telluric lines can be defined at high signal-tonoise $(\mathrm{S} / \mathrm{N})$ and in a short integration time. The stellar spectrum in itself has very little influence on the obtained RV; the only eventual issue is the blending between stellar and atmospheric lines.

\section{Methodology and data reduction}

A dedicated pipeline was created to reduce HARPS spectra, called Data Reduction Software hereafter DRS. For more details we refer to Mayor et al. (2003). In a nutshell, the pipeline provides the typical bad pixel/column, bias, and dark correction, flat-fielding, and optimal extraction of the spectra. The spectra are then calibrated in wavelength using a Th-Ar lamp. The wavelength-calibrated spectra are cross-correlated (as described in Baranne et al. 1996) with a weighted template mask (Pepe et al. 2002). To calculate the RV of the atmospheric lines in the spectra with the existing pipeline one then needs to construct a telluric mask.

Using the HITRAN database (Rothman et al. 2005), one can locate 11992 spectral lines within the HARPS wavelength domain. These lines correspond to $\mathrm{H}_{2} \mathrm{O}, \mathrm{OH}$, and $\mathrm{O}_{2}$ molecules (with 8706, 3060, and 226 lines, respectively) and cover mainly the red side of the spectra, from 540 to $690 \mathrm{~nm}$. Water lines span the widest wavelength range, but are of a very high dynamic range and are strongly blended. Most of the $\mathrm{OH}$ lines are too faint to be detected and are not even visible in Delbouilles FTS spectrum. Out of the 226 oxygen absorption features, one can find 71 lines deeper than $1 \%$ and some as deep as $95 \%$. These lines are distributed in 2 bands, located at 628-663 and $687-690 \mathrm{~nm}$. The features are well-separated spectrally by HARPS, exhibiting no clumping neither among themselves nor with stellar lines, unlike the other two species. Following the arguments we have presented, we compiled a mask of only $\mathrm{O}_{2}$ lines. The vacuum wavelengths were drawn from the HITRAN database, and the corresponding values at standard temperature and pressure were calculated. The depth of each line could not be estimated accurately by fitting Gaussian functions to the high $\mathrm{S} / \mathrm{N}$ spectra; for deep lines $(>90 \%)$ the departure from Gaussian profile was too pronounced and the errors resulting from this assumption were non-negligible. As an alternative we used the Delbouille FTS spectra ${ }^{1}$. This high-resolution spectra was convolved with an instrumental profile representative of HARPS resolution and the resulting spectra were analyzed. The depth of each line was then estimated by measuring the depth of the synthetic absorption line. The depth of the corresponding spectra was found to differ by only $1-2 \%$ relative to the normalized observed spectra depth. The objective of this procedure was to assign the correct depth and thus the correct weight to each line when calculating the cross-correlation, as described in Pepe et al. (2002).

\footnotetext{
1 The original reference is http://cdsads.u-strasbg.fr/ abs/1973apds.book.....D, and the data are also available from the BASS 2000 database at http://bass2000.obspm.fr/ solar_spect.php in a much easier way to use.
} 


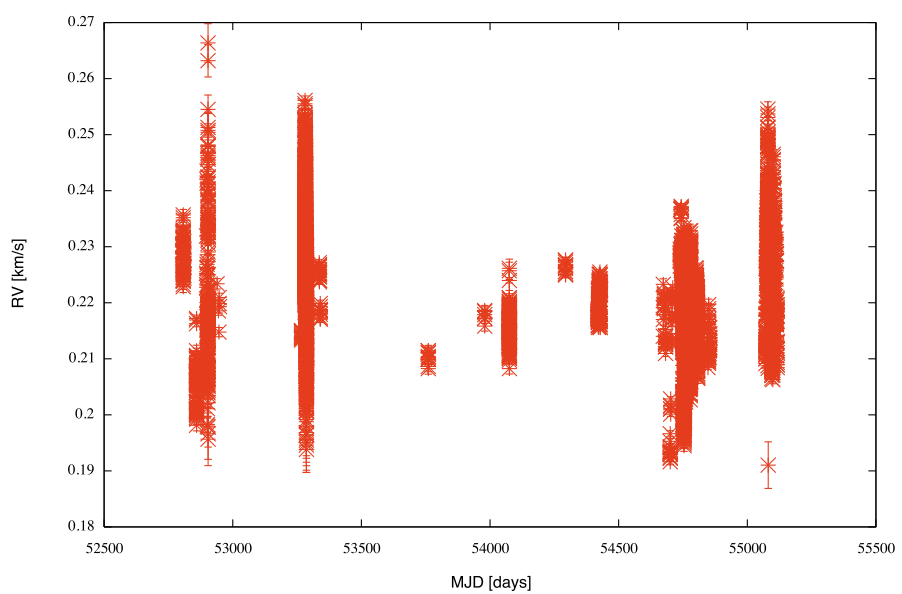

Fig. 1. The telluric RV variation in all Tau ceti measurements as a function of time.

Table 2. The dispersion and average photon noise of the stars used in our campaign.

\begin{tabular}{lcc}
\hline \hline \multicolumn{1}{c}{ Target } & $\sigma[\mathrm{m} / \mathrm{s}]$ & $\sigma_{\mathrm{ph}}[\mathrm{m} / \mathrm{s}]$ \\
\hline Tau Ceti & 10.74 & 0.98 \\
$\mu$ Ara & 10.31 & 1.35 \\
$e$ Eri & 10.82 & 0.76 \\
\hline
\end{tabular}

The pipeline measures the RV and the associated photon noise error, by analyzing the CCF function as described in Bouchy et al. (2001). In addition to each RV calculation, the bisector inverse slope was computed from the CCF function following the procedure described in Queloz et al. (2001).

\section{Results and analysis}

From our analysis we excluded spectra which delivered a very low photon noise precision, superior to $5 \mathrm{~m} / \mathrm{s}$. These correspond to a very small fraction of the measurements, $0.8 \%$ for Tau Ceti, $1.08 \%$ for $\mu$ Ara, and $0.2 \%$ for $e$ Eri.

The scatter in the RV measurements and the average photon noise for each star are presented in Table 2. The RV variations are very low, roughly $10 \mathrm{~m} / \mathrm{s}$ for all three stars over 6 years. We plot the RV of Tau Ceti as a function of time in Fig. 1. The low scatter is a remarkable result, especially if we consider that no modeling, correction, or filtering were involved. Nevertheless, the dispersion is much higher than the photon noise, which indicates that there is an additional source in the measured scatter.

We note that even data subsets covering a short timescale interval, e.g. one night, already exhibit an RV variation larger than the average photon noise. This infers that the measured RV depends on a factor that varies at a timescale of one night. In Fig. 2, we present the atmospheric lines RV variation in the spectra of Tau Ceti over a full night (upper left panel), which clearly illustrates this point.

In this series of measurements the BIS of the CCF clearly varies throughout the night (lower left panel). The BIS seems to be linearly correlated with both the airmass (upper right panel) and the FWHM of the fitted CCF (lower right panel). We omit from this series of plots one showing the correlation between contrast and airmass, which resembles the correlation between FWHM and airmass. Observing a star at different airmasses clearly produces different absorption features, with contrast and FWHM that depend linearly on airmass. The line profile variation is also asymmetric, as the BIS plot ilustrates, and scales with airmass. This asymmetry is therefore expected to have an impact on the measured RV.

These aforementioned properties are consistent with some molecular lines being naturally asymmetric (e.g., Frommhold 2006) $)^{2}$. In observations at different airmasses, the light passes through a medium with different path lengths. Because absorption does not vary linearly with geometric path length but exponentially, the line shape is bound to change. When one observes at higher airmass, the absorption introduced by the additional molecules is stronger on the wings than in the core, producing a larger, deeper, and more asymmetric absorption line. We note that the CCF contrast oscillates around $72 \%$, and many lines have a contrast greater than $90 \%$. These lines are no longer in the linear regime. Another possible explanation is that as the line broadening increases with pressure, the asymmetry becomes detectable. This was measured for $\mathrm{H}_{2}$ in the Solar System gaseous planets by Cochran \& Smith (1983).

However, as seen on Fig. 2, this effect cannot alone explain the shape drawn by the RV, and another effect that operates at a timescale of one night must be present. From our previous discussion about the airmass/bisector influence, this second effect does not have an impact on the line asymmetry; it must therefore shift the center of atmospheric lines.

One possible explanation is a predominant, horizontal wind, constant during the night. As one follows a star across the sky, the projection of the wind vector along the line of sight of the telescope changes. For a given telescope elevation $\theta$ and azimuth $\phi$, the projection of an horizontal wind with direction $\delta$ and speed $w_{\mathrm{s}}$ along the line of sight of the telescope is given by

$w_{\mathrm{s}, \operatorname{proj}}=w_{\mathrm{s}} \times \cos (\theta) \times \cos (\phi-\delta)$.

Unfortunately, both these parameters are unknown ${ }^{3}$. Given the high number of points and good sampling of the parameters space, one can try to fit the mentioned effects. The measured RV is expected to be proportional to 1) airmass and 2) wind velocity projected along the line of sight. This corresponds to determine $\alpha, \beta$, and $\delta$ such that the quantity

$\Omega=\alpha \times\left(\frac{1}{\sin (\theta)}-1\right)+\beta \times \cos (\theta) \times \cos (\phi-\delta)+\gamma$

is linearly correlated with the measured RV (note that we used the fact that airmass $=\sin ^{-1}(\theta)$ ). The $\gamma$ represents the zero-point of the RV. This quantity may differe from zero $\mathrm{m} / \mathrm{s}$, because of an error in the line's vacuum wavelength or in the wavelength conversion from vacuum to air.

We used a weighted linear least squares minimization to determine the best fit parameters for each subset of data. This approach was employed for subsets corresponding to complete nights, for which the hypothesis of a constant wind is a reasonable one. We compared the results yielded by linear least squares with that of non-linear least squares (using a Levenberg-Marquardt algorithm) and we recovered the same results.

\footnotetext{
${ }^{2}$ Note that this is the case for homonuclear diatomic molecules, like $\mathrm{O}_{2}$.

3 The weather stations at La Silla monitor the wind at atmospheric ground layer. It is well known that the wind at the ground layer is detached from those of high altitude, and as a consequence we cannot use weather monitor values as a proxy for either $w_{\mathrm{s}}$ or $\delta$.
} 

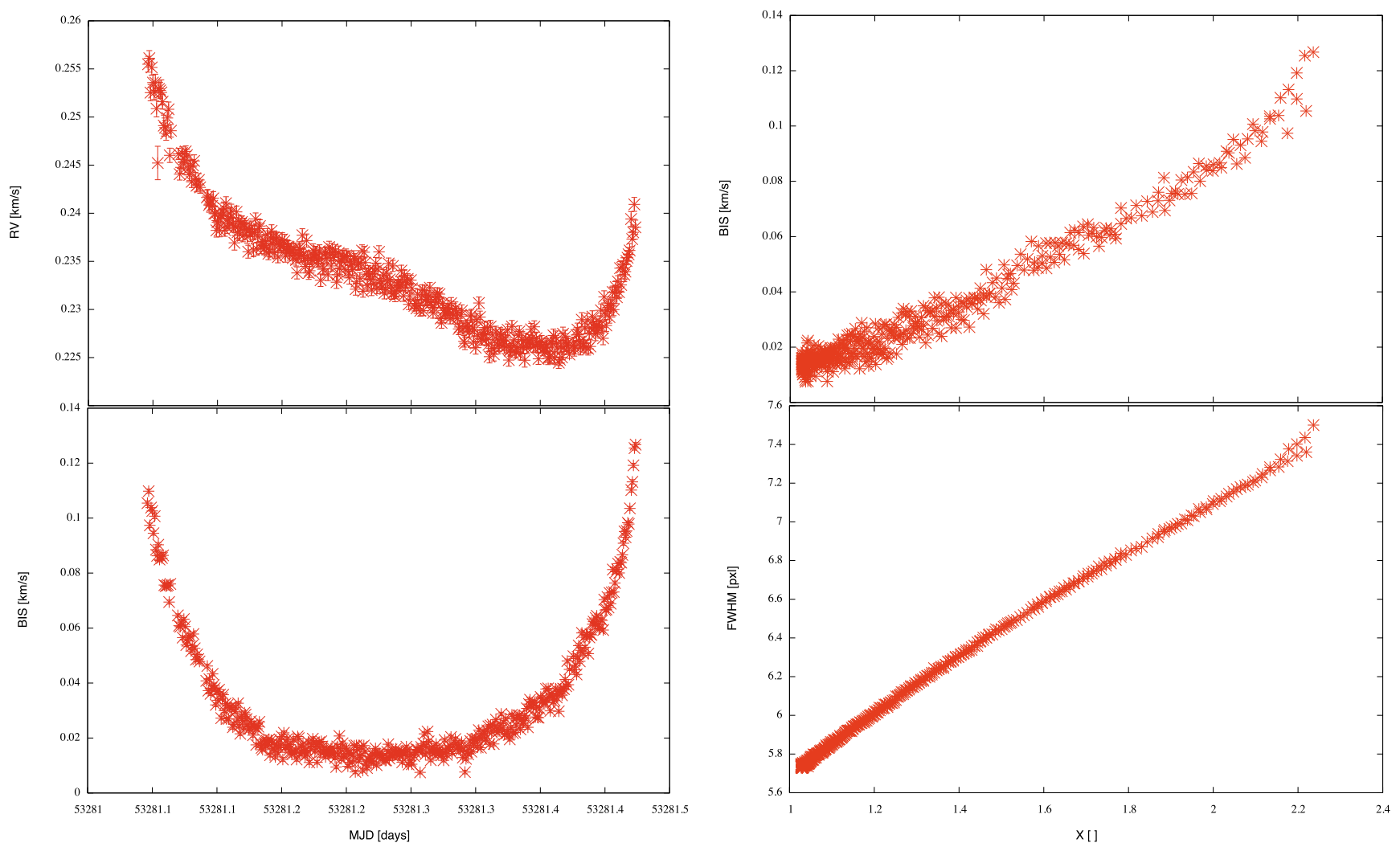

Fig. 2. Telluric RV measurements on Tau Ceti over a full night. Note the clear shape drawn by the RV (left panel, top) and the associated bisector (left panel, bottom) as function of time. In the right panel we depict the correlation between BIS and airmass (right panel, top) and FWHM and airmass (right panel, bottom). The plotted errorbars in RV and BIS correspond to photon errors. Photon errors in the BIS are approximated to be twice the RV errors.

Table 3. The fitted parameters and data properties, before and after the fitted model is subtracted from it.

\begin{tabular}{ccccccccccc}
\hline \hline Target & Data set & \#obs & $\sigma[\mathrm{m} / \mathrm{s}]$ & $\sigma_{(\mathrm{O}-\mathrm{C})}[\mathrm{m} / \mathrm{s}]$ & $\sigma_{\mathrm{ph}}[\mathrm{m} / \mathrm{s}]$ & $\chi_{\text {red }}^{2}$ & $\alpha[\mathrm{m} / \mathrm{s}]$ & $\beta[\mathrm{m} / \mathrm{s}]$ & $\gamma[\mathrm{m} / \mathrm{s}]$ & $\delta\left[{ }^{\circ}\right]$ \\
\hline Tau Ceti & $2004-10-03$ & 437 & 6.40 & 1.67 & 0.64 & $\dagger$ & 17.75 & 43.39 & 222.01 & -167.21 \\
& $2004-10-04$ & 438 & 7.98 & 1.33 & 0.65 & $\dagger$ & - & 27.89 & - & -154.15 \\
& $2004-10-05$ & 599 & 7.12 & 2.03 & 0.79 & $\dagger$ & - & 15.17 & - & -133.95 \\
\hline$\mu$ Ara & $2004-06-04$ & 278 & 6.90 & 1.90 & 1.27 & $\dagger$ & - & 33.27 & - & -155.37 \\
& $2004-06-05$ & 274 & 8.35 & 2.50 & 1.30 & $\dagger$ & - & 29.34 & - & -140.20 \\
& $2004-06-06$ & 285 & 8.94 & 1.72 & 1.11 & $\dagger$ & - & 27.45 & - & -136.20 \\
& $2004-06-07$ & 286 & 4.48 & 1.60 & 1.03 & $\dagger$ & - & 23.62 & - & -165.43 \\
& $2004-06-08$ & 275 & 3.98 & 1.81 & 1.07 & $\dagger$ & - & 36.61 & - & -168.70 \\
& $2004-06-09$ & 214 & 6.88 & 4.02 & 1.34 & $\dagger$ & - & 41.89 & - & -164.93 \\
& $2004-06-10$ & 202 & 6.92 & 2.55 & 1.81 & $\dagger$ & - & 41.74 & - & -142.11 \\
& $2004-06-11$ & 273 & 8.41 & 3.51 & 2.07 & $\dagger$ & - & 48.87 & - & -155.55 \\
\hline Both stars & all data & 3562 & 11.79 & 2.27 & 1.09 & 4.01 & & & & \\
\hline
\end{tabular}

Notes. In this fit, $\alpha$ and $\gamma$ are imposed to be the same for all data sets. Since the fit is made simultaneously for all data sets, the $\chi_{\text {red }}^{2}$ calculation is not applicable for a single night and the respective table entries are indicated by a $\uparrow$. The table structure is left unchanged to allow for an easier comparison with Tables A.1 and A.2. Note that $\delta=0$ corresponds to the south-north direction.

If Eq. (2) correctly describes the phenomena affecting the $\mathrm{RVs}$, then the parameter $\gamma$ is constant with time, by construction. From the same equation we conclude that this parameter is simply the RV measured at zenith, where the airmass and wind projection effects are null. One can then fit the different data sets while imposing the same $\gamma$ for all. By the same reasoning, $\alpha$ can be fixed. This is justified by the aforementioned assumption that the bisector effect is caused by the fundamental asymmetry of line and thus should not vary with time.
The results of the fitting are presented in Table 3 . We present the fitted parameters, along with the dispersion before and after the fitted function is subtracted from the data, and the $\chi_{\text {red }}^{2}$ for each fit.

We also considered the cases in which $\alpha$ and both $\alpha$ and $\gamma$ are allowed to vary. The results are presented in Appendix A in Tables A.1 and A.2, respectively.

The low chi-squared, within the range 2.5-4.01, attests to the validity of the empirical description of the $\mathrm{RV}$ variation. The 


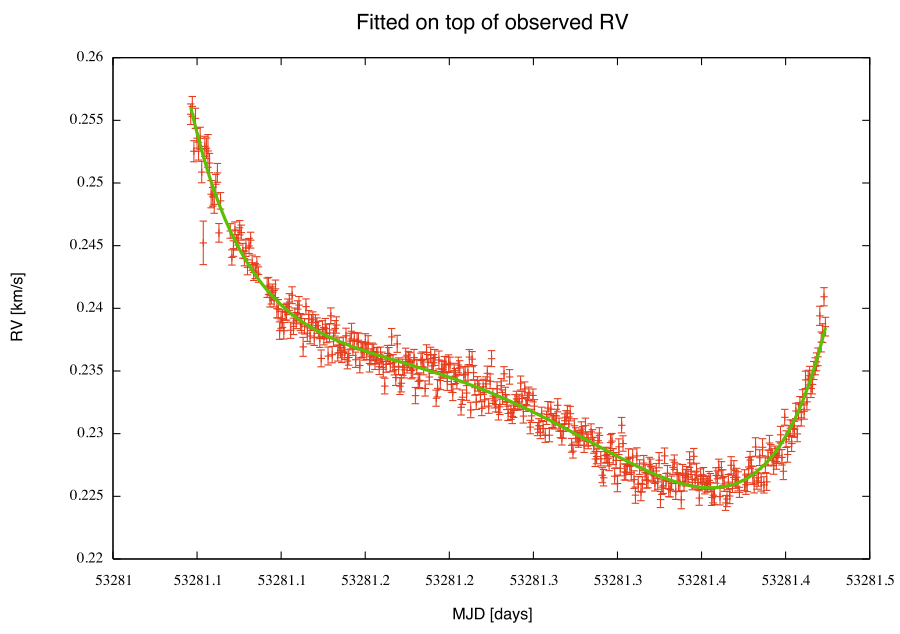

Fig. 3. The fit of the atmospheric lines RV variation for the first night of the asteroseismology run of Tau Ceti. The fitted model is described by Eq. (2) and the parameters are presented in Table A.1.

residuals around the fit are about twice the photon noise, showing that this correction is effective down to roughly $2 \mathrm{~m} / \mathrm{s}$. As an example, the fit to the data set corresponding to the first night of the Tau Ceti campaign is presented in Fig. 3.

\section{Discussion}

We have demonstrated that $\mathrm{O}_{2}$ telluric lines are stable to $10 \mathrm{~m} / \mathrm{s}$ (rms) on a timescale of 6 years. This is despite atmospheric phenomena, which introduce variations at the $1-10 \mathrm{~m} / \mathrm{s}$ level, and can be corrected using simple atmospheric models, as described in Sect. 4.

The properties of the fitted parameters are very insightful in themselves. The imposition of a constant $\alpha$ and $\gamma$ for the different data sets lead to $\beta$ and $\delta$ values that vary more smoothly over time and are thus more likely to correspond to the physical ones. We would expect precisely the opposite if the model assumptions were wrong because as the number of parameters were reduced, the remaining ones would have to vary more to compensate for the observed variations. We conclude therefore that the description of the phenomena is correct. We cannot, however, discard that our model cannot be refined. For instance, the airmass effect might depend on a second order on the wind speed. In any case, the present model provides good results to twice the photon noise level. Allowing all the fitting parameters to vary reduces the $\chi_{\text {red }}^{2}$, but not in a very significant way; we thus consider the approach of fixing the $\alpha$ and $\gamma$ to be the most adequate.

The fitting delivers commonly $\beta$ larger than $\alpha$, i.e., the effect of wind projection on RV is usually of higher magnitude than the airmass effect. We note that the wind direction is predominantly from between $\mathrm{N}$ and $\mathrm{NE}$ and roughly of $40 \mathrm{~m} / \mathrm{s}$, which is a common value for high-altitude winds over La Silla.

The constancy of the wind over a short time interval and that the two effects scale with airmass attests that telluric lines can be straightforwardly used as a precise wavelength reference over short time scales, if one observes at low airmass. In Table 4, we present the dispersion in RV on timescales of 1-8 days at restricted airmass, quantifying this conclusion. A precision of $5 \mathrm{~m} / \mathrm{s}$ can then clearly be routinely obtained without using any atmospheric corrections if one observes in these conditions.
Table 4. The measured dispersion for data-sets with restricted airmass, for time-spans of 1-8 days and the whole data-set.

\begin{tabular}{ccccc}
\hline \hline Target & Data-set $[\mathrm{d}]$ & $\sigma_{(X<1.5)}$ & $\sigma_{(X<1.2)}$ & $\sigma_{(X<1.1)}[\mathrm{m} / \mathrm{s}]$ \\
\hline Tau Ceti & 1 & 4.53 & 3.38 & 2.41 \\
& 2 & 5.53 & 4.07 & 3.27 \\
& 3 & 5.81 & 4.55 & 3.70 \\
& Full & 9.77 & 9.16 & 8.43 \\
\hline$\mu$ Ara & 1 & 5.00 & 3.36 & 2.31 \\
& 2 & 6.18 & 4.14 & 2.61 \\
& 3 & 6.38 & 4.27 & 2.66 \\
& 4 & 5.67 & 3.86 & 2.39 \\
& 5 & 5.67 & 4.01 & 2.57 \\
& 6 & 6.18 & 4.71 & 3.24 \\
& 7 & 6.46 & 4.93 & 3.40 \\
& 8 & 6.94 & 5.42 & 3.91 \\
$\epsilon$ Eri & Full & 9.88 & 10.10 & 10.23 \\
\hline
\end{tabular}

We recall that both $\mathrm{O}_{2}$ and $\mathrm{CO}_{2}$ are abundant species in the atmosphere, with a roughly constant v.m.r. up to $80 \mathrm{~km}$. This implies that these molecules are less sensitive to short timescale weather variations and the assumptions of a constant horizontal wind are realistic hypothesis.

We have not checked whether the correlation with our mask is affected by blending between telluric and stellar lines. The superposition of stellar lines on the telluric spectra varies from star to star, due to the different mean stellar RV and the projection of Earth's motion around the Sun along the line of sight to the star. However, for this to bias the final RV, i.e., introduce a systematic error, the blends would have to be similar for all lines. As a consequence, the superposition of stellar and telluric lines will, if present, only lead to an increase in the scatter of the measured RVs. An extensive study has been initiated to understand the impact of this effect.

\section{Conclusions}

Our results have proven that $\mathrm{O}_{2}$ telluric lines are stable down to $10 \mathrm{~m} / \mathrm{s}$ on a timescale of 6 years. Atmospheric phenomena introduce variations at the $1-10 \mathrm{~m} / \mathrm{s}$ level (rms). These can be accounted for by simple atmospheric models, which allow us to reach a precision of $2 \mathrm{~m} / \mathrm{s}$, twice the photon noise.

This work has illustrated the power of atmospheric features as a wavelength calibrator. It is now clear that this approach can compete with gas-cell methods on short timescales (of 1 week) in a straightforward way. A better precision than gas cell can even be achievable on longer timescales if the atmospheric variations are characterized and compensated for by simple modeling.

Acknowledgements. Support from the Fundação para Ciência e a Tecnologia (Portugal) to P. F. in the form of a scholarship (reference SFRH/BD/21502/2005) is gratefully acknowledged. P.F. thanks to everyone who contributed to Planetary group meetings, and the referee Bill Cochran for his valuable comments on the paper.

\section{Appendix A: Aditional fitting results}

In this section we present the parameters for the best fit of Eq. (2), the dispersion of the data before and after the fit 
A\&A 515, A106 (2010)

Table A.1. The fitted parameters and data properties, before and after the fitted model is subtracted from it, when all parameters are left to vary freely.

\begin{tabular}{ccccccccccc}
\hline \hline Target & Dataset & $\#$ obs & $\sigma[\mathrm{m} / \mathrm{s}]$ & $\sigma_{(\mathrm{O}-\mathrm{C})}[\mathrm{m} / \mathrm{s}]$ & $\sigma_{\mathrm{ph}}[\mathrm{m} / \mathrm{s}]$ & $\chi_{\text {red }}^{2}$ & $\alpha[\mathrm{m} / \mathrm{s}]$ & $\beta[\mathrm{m} / \mathrm{s}]$ & $\gamma[\mathrm{m} / \mathrm{s}]$ & $\delta\left[{ }^{\circ}\right]$ \\
\hline Tau Ceti & $2004-10-03$ & 437 & 6.40 & 1.05 & 0.64 & 2.52 & 32.34 & 83.91 & 212.53 & -173.24 \\
& $2004-10-04$ & 438 & 7.98 & 1.16 & 0.65 & 3.05 & 27.84 & 60.45 & 214.36 & -168.28 \\
& $2004-10-05$ & 599 & 7.12 & 1.40 & 0.79 & 3.33 & 32.75 & 75.65 & 208.01 & -171.96 \\
\hline$\mu$ Ara & $2004-06-04$ & 278 & 6.90 & 1.75 & 1.27 & 1.92 & 24.31 & 64.66 & 235.34 & -167.43 \\
& $2004-06-05$ & 274 & 8.35 & 2.13 & 1.30 & 2.15 & 1.39 & 27.65 & 207.39 & -43.82 \\
& $2004-06-06$ & 285 & 8.94 & 1.69 & 1.11 & 2.15 & 23.61 & 43.38 & 229.04 & -154.23 \\
& $2004-06-07$ & 286 & 4.48 & 1.58 & 1.03 & 2.39 & 24.81 & 46.31 & 230.45 & -172.64 \\
& $2004-06-08$ & 275 & 3.98 & 1.63 & 1.07 & 2.35 & 32.92 & 100.02 & 246.72 & -175.53 \\
& $2004-06-09$ & 214 & 6.88 & 3.36 & 1.34 & 2.63 & 34.04 & 121.93 & 252.77 & -172.50 \\
& $2004-06-10$ & 202 & 6.92 & 2.36 & 1.81 & 1.74 & -5.26 & 47.60 & 195.54 & -33.05 \\
& $2004-06-11$ & 273 & 8.41 & 3.48 & 2.07 & 2.24 & 27.72 & 81.03 & 234.53 & -165.52 \\
\hline Both stars & all data & 3562 & 11.79 & 1.95 & 1.09 & 2.52 & & & & \\
\hline
\end{tabular}

Table A.2. The fitted parameters and data properties, before and after the fitted model is subtracted from it, with the $\gamma$ imposed to be the same for all datasets.

\begin{tabular}{ccccccccccc}
\hline \hline Target & Dataset & \#obs & $\sigma[\mathrm{m} / \mathrm{s}]$ & $\sigma_{(\mathrm{O}-\mathrm{C})}[\mathrm{m} / \mathrm{s}]$ & $\sigma_{\mathrm{ph}}[\mathrm{m} / \mathrm{s}]$ & $\chi_{\text {red }}^{2}$ & $\alpha[\mathrm{m} / \mathrm{s}]$ & $\beta[\mathrm{m} / \mathrm{s}]$ & $\gamma[\mathrm{m} / \mathrm{s}]$ & $\delta\left[{ }^{\circ}\right]$ \\
\hline Tau Ceti & $2004-10-03$ & 437 & 6.40 & 1.05 & 0.64 & $\dagger$ & 31.62 & 81.42 & 213.09 & -173.03 \\
& $2004-10-04$ & 438 & 7.98 & 1.17 & 0.65 & $\dagger$ & 29.44 & 66.03 & - & -169.28 \\
& $2004-10-05$ & 599 & 7.12 & 1.47 & 0.79 & $\dagger$ & 26.33 & 53.16 & - & -168.52 \\
\hline$\mu$ Ara & $2004-06-04$ & 278 & 6.90 & 1.90 & 1.27 & $\dagger$ & 8.52 & 15.11 & - & -106.45 \\
& $2004-06-05$ & 274 & 8.35 & 2.15 & 1.30 & $\dagger$ & 5.81 & 19.69 & - & -76.20 \\
& $2004-06-06$ & 285 & 8.94 & 1.78 & 1.11 & $\dagger$ & 11.59 & 19.42 & - & -78.89 \\
& $2004-06-07$ & 286 & 4.48 & 1.67 & 1.03 & $\dagger$ & 11.53 & 5.91 & - & -83.57 \\
& $2004-06-08$ & 275 & 3.98 & 1.86 & 1.07 & $\dagger$ & 7.95 & 12.34 & - & -142.69 \\
& $2004-06-09$ & 214 & 6.88 & 3.91 & 1.34 & $\dagger$ & 7.50 & 19.43 & - & -139.67 \\
& $2004-06-10$ & 202 & 6.92 & 2.41 & 1.81 & $\dagger$ & 8.04 & 26.12 & - & -107.04 \\
& $2004-06-11$ & 273 & 8.41 & 3.59 & 2.07 & $\dagger$ & 12.27 & 29.56 & - & -136.75 \\
\hline Both stars & all data & 3562 & 11.79 & 2.08 & 1.09 & 2.80 & & & & \\
\hline
\end{tabular}

Notes. Since the fit is made simultaneously for all datasets, the $\chi_{\text {red }}^{2}$ calculation is not applicable for a single night and the respective table entries are signaled by a $\uparrow$.

correction, the average photon noise and the $\chi_{\text {red }}^{2}$. Note that $\delta=0$ corresponds to the South-North direction.

\section{References}

Balthasar, H., Thiele, U., \& Woehl, H. 1982, A\&A, 114, 357 Baranne, A., Queloz, D., Mayor, M., et al. 1996, A\&AS, 119, 373 Bouchy, F., Pepe, F., \& Queloz, D. 2001, A\&A, 374, 733

Butler, R. P., Marcy, G. W., Williams, E., et al. 1996, PASP, 108, 500

Caccin, B., Cavallini, F., Ceppatelli, G., Righini, A., \& Sambuco, A. M. 1985, A\&A, 149, 357

Cochran, W. D., \& Smith, W. H. 1983, ApJ, 271, 859

Figueira, P., Pepe, F., Melo, C. H. F., et al. 2010, A\&A, 511, A55
Frommhold, L. 2006, Collision-Induced Absorption in Gases, ed. L. Frommhold Griffin, R., \& Griffin, R. 1973, MNRAS, 162, 255

Huélamo, N., Figueira, P., Bonfils, X., et al. 2008, A\&A, 489, L9

Lovis, C., Mayor, M., Pepe, F., et al. 2006, Nature, 441, 305

Lovis, C., \& Pepe, F. 2007, A\&A, 468, 1115

Mayor, M., \& Queloz, D. 1995, Nature, 378, 355

Mayor, M., Pepe, F., Queloz, D., et al. 2003, The Messenger, 114, 20

Mayor, M., Udry, S., Lovis, C., et al. 2009, A\&A, 493, 639

Pepe, F., Mayor, M., Galland, F., et al. 2002, A\&A, 388, 632

Queloz, D., Henry, G. W., Sivan, J. P., et al. 2001, A\&A, 379, 279

Rothman, L. S., Jacquemart, D., Barbe, A., et al. 2005, J. Quant. Spectrosc.

Radiat. Transfer, 96, 139

Smith, M. A. 1982, ApJ, 253, 727

Snellen, I. A. G. 2004, MNRAS, 353, L1 